\title{
Rare earth substitutional impurities in germanium: a hybrid density functional theory study
}

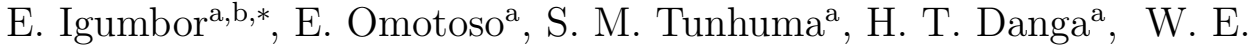 \\ Meyer $^{\mathrm{a}, * *}$ \\ ${ }^{a}$ Department of Physics, University of Pretoria, Pretoria 0002, South Africa. \\ ${ }^{b}$ Department of Mathematics and Physical Sciences, Samuel Adegboyega University, Km \\ 1 Ogwa/Ehor Rd, Ogwa Edo State Nigeria.
}

\section{Abstract}

The Heyd, Scuseria, and Ernzerhof (HSE06) hybrid functional by means of density functional theory has been used to modelled the electronic and structural properties of rare earth (RE) substitutional impurities in germanium $\left(\mathrm{RE}_{\mathrm{Ge}}\right)$. The formation and charge state transition energies for the $\mathrm{RE}_{\mathrm{Ge}}(\mathrm{RE}=\mathrm{Ce}, \mathrm{Pr}, \mathrm{Er}$ and $\mathrm{Eu})$ were calculated. The energy of formation for the neutral charge state of the $\mathrm{RE}_{\mathrm{Ge}}$ lies between -0.14 and $3.13 \mathrm{eV}$. The formation energy result shows that the $\operatorname{Pr}$ dopant in $\operatorname{Ge}\left(\operatorname{Pr}_{\mathrm{Ge}}\right)$ has the lowest formation energy of $-0.14 \mathrm{eV}$, and is most energetically favourable under equilibrium conditions. The $\mathrm{RE}_{\mathrm{Ge}}$ induced charge state transition levels within the band gap of Ge. Shallow acceptor levels were induced by both the $\mathrm{Eu}\left(\mathrm{Eu}_{\mathrm{Ge}}\right)$ and $\operatorname{Pr}\left(\mathrm{Pr}_{\mathrm{Ge}}\right)$ dopants in Ge. The $\mathrm{Ce}_{\mathrm{Ge}}$ and $\mathrm{Er}_{\mathrm{Ge}}$ exhibited properties of negative- $U$ ordering with effective- $U$ values of -0.85 and $-1.07 \mathrm{eV}$, respectively.

\footnotetext{
${ }^{*}$ Corresponding author

${ }^{* *}$ Corresponding author

Email addresses: elgumuk@gmail.com (E. Igumbor), wmeyer@up.ac.za (W. E. Meyer )
} 
Keywords: Defects, formation energy, charge state, rare earth

\section{Introduction}

Germanium (Ge) is being considered as a material for new generation of microelectronic devices, as a result of its unique properties (carrier mobilities, low dopant activation temperatures and smaller band-gap of $0.78 \mathrm{eV}$ at 0 Kelvin) $[1,2,3]$. Several studies of point defects in Ge using experimental [4, $5,6,7]$ or theoretical $[8,9,10,11,12]$ techniques have been reported. It has been reported that defect processes of dopants in Ge semiconductors can be influenced by doping and double-donor doping, which may be an effective way to engineer the active donor concentrations of Ge [9]. Rare earth (RE) impurities in semiconductor material have attracted interest due to their unique predictable optical properties [13]. Another interesting property of the RE is its ability to participate in photoluminescence process. For instance, the $\mathrm{Er}^{3+}$ ions have been discovered to participate in an energy transfer process during optical or electrical pumping of Er-doped $\mathrm{SiO}_{2}$ [14]. In another development, according to report by Kanjilal et al [15], the Erdoped amorphized Ge nanoclusters can recrystallized in absence of Ge outdiffusion during flash-lamp annealing. Channelling experiments by Jones et at [16] have given support for a Er tetrahedral interstitial location in Si. Visible emission as well as an infrared $1.53 \mu \mathrm{m}$ signal from the Er doped Ge nanowires at room temperature have been observed [17]. Recently, Tm [10] substitutional impurity in Ge was predicted to induce in the band gap of Ge an acceptor level $(-1 /-2)$ at $E_{C}-0.05 \mathrm{eV}\left(E_{C}\right.$ and $E_{V}$ are the energies of the conduction band minimum and the valence band maximum respectively), and 
double donor levels at $E_{V}+0.10$ and $E_{V}+0.19$ for the $(+2 /+1)$ and $(+1 / 0)$ charge state transitions, respectively. The interaction energy between two electrons in a two-level defect is referred to as Hubbard- $U$ [18, 19]. Hubbard$U$ is regarded as a negative- $U$ when the neutral charge state is excited, and it becomes energetically less favourable. A good number of defects in Ge and Si have been observed to exhibit charge state negative- $U$ ordering $[2,10,20]$. The $\mathrm{Tm}^{3+}$ and $\mathrm{Ce}^{3+}$ interstitials in Ge are found to exhibit charge state negative- $U$ ordering $[20,10]$.

It has been reported that materials in the future (due to miniaturization) will have shallow junctions with high conductivity [21, 22]. For Ge based device processing damage occurs during ion implantation. As a result, the role of defects that enhance diffusion of the implanted dopant will have to be well understood. Bracht et al [7] in a review reported the mechanism of selfand dopant diffusion in Ge under thermal equilibrium and non-equilibrium conditions. In this case, the non-equilibrium conditions can be realized by irradiation or implantation. While $\mathrm{RE}$ ion defects in $\mathrm{Si}$ and other materials have been studied either by theoretical modelling or by experimental techniques $[23,24,25]$. Except for the Tm [10], the structural and electronic properties of RE substitutional defects in Ge have not been theoretically studied. In order for us to provide a frontier insight for the experimental study of the electronic properties of defects resulting from the implantation of the RE in Ge, we have used the hybrid functional of Heyd, Scuseria, and Ernzerhof (HSE06) [26] by means of density functional theory (DFT) to calculate the structural and electronic properties of rare earth substitutions in $\mathrm{Ge}\left(\mathrm{RE}_{\mathrm{Ge}}\right.$ for RE: Ce, Pr, Eu and Er). The energies of formation for the $\mathrm{Ce}_{\mathrm{Ge}}$, 
$\operatorname{Pr}_{\mathrm{Ge}}, \mathrm{Eu}_{\mathrm{Ge}}$ and $\mathrm{Er}_{\mathrm{Ge}}$ are calculated for charge states $(-2,-1,0,+1,+2)$. The charge state transition levels with the accessible thermodynamic region are examined and presented as well.

\section{Computational details}

Results of this report are based on DFT. We used the projector-augmented wave (PAW) $[27,28]$ to separate the core electrons from the valence electrons. Vienna Ab initio Simulation Package (VASP) [27] code was used. All calculations were carried out using Heyd, Scuseria, and Ernzerhof (HSE06) [26] hybrid functional. According to the HSE06 approach, the short-range exchange potential is calculated by mixing $25 \%$ fraction of nonlocal HartreeFock exchange with the Perdew, Burke, and Ernzerhof (PBE) [29] functional. In the past, defects in Ge were difficult to study theoretically, since the local density approximation (LDA) and the generalized gradient approximation (GGA) functionals incorrectly predict Ge to be a metal $[2,30]$. However, the HSE06 functional has been used to predict accurately the electronic band gap and improve charge state transition properties for group-IV semicon-

ductors $[2,12,30]$. According to our previous reports [10, 20], the modelling and prediction of the electronic properties of material with the $f$ orbital valence shell was difficult, because the $f$ orbital is highly localized. Recently, the hybrid functional has been successfully used to predict the electronic and band gap properties of several materials with the $f$ orbital in the valence shell [10,31, 32]. Following this success of the hybrid functional, the $f$ orbital in the valence shell of RE can be handle.

To calculate the properties of the RE substitutional impurities in Ge, a 
periodic supercell containing 64 atoms of Ge was used. A regularly spaced mesh of $2 \times 2 \times 2$ Monkhorst-Pack [33] scheme k-point was used to integrate the Brillouin zone. We set the plane wave cutoff of the wave function expansion to $400 \mathrm{eV}$. To obtain an equilibrium geometry of a perfect supercell, we refined the geometry until the final change in the total energy and the forces were less than $10^{-5} \mathrm{eV}$ and $0.001 \mathrm{eV} / \AA$, respectively. The formation energy of a strongly correlated system is strongly dependent on the spin-orbit coupling (SOC) due to the presence of relativistic effects in heavy atomic systems. The scalar relativistic effect was taken into consideration by including in the PAW potential the mass - velocity and Darwin correction terms. In addition, spin orbit coupling was taken into account for all calculations. The formation energy $\left(E^{f}\right)$ of defects is derived directly from total energies, allowing the calculation of equilibrium defect concentrations [34]. To determine the defect formation energy and transition energy $\left(\epsilon\left(q / q^{\prime}\right)\right)$ levels, we calculated the total energy $\mathrm{E}\left(\mathrm{RE}_{\mathrm{Ge}}, q\right)$ for a supercell containing an optimized defect $\mathrm{RE}_{\mathrm{Ge}}$ in its charge state $q$. The RE substitutional impurity in Ge formation energy $E^{f}\left(\mathrm{RE}_{\mathrm{Ge}}, q\right)$ as a function of electron Fermi energy $\left(\varepsilon_{F}\right)$ is given as [35]

$E^{f}\left(\mathrm{RE}_{\mathrm{Ge}}, q\right)=E\left(\mathrm{RE}_{\mathrm{Ge}}, q\right)-E($ pristine $)+\sum_{i}(\triangle n)_{i} \mu_{i}+q\left[E_{V}+\varepsilon_{F}\right]+E_{c o r}^{q}$,

where E(pristine) is the total energy of a supercell containing 64 atoms of Ge, $(\triangle n)_{i}(\triangle(n)<0$, when a RE atom is included to a supercell containing 64 atoms of Ge and $\triangle(n)>0$ when a Ge atom is removed from a supercell containing 64 atoms of $\mathrm{Ge}$ ) is the difference in the number of constituent atoms of type $i$ between the supercell containing 64 atoms of Ge and the supercell containing the defect, $\mu_{i}$ represents the chemical potential of differ- 
ent constituent atoms and as mentioned earlier, $\mathrm{E}_{\mathrm{V}}$ is energy of the valence band maximum (VBM). The $\mathrm{E}_{\mathrm{cor}}^{\mathrm{q}}$ is the Freysoldt, Neugebauer, and Van de Walle $(\mathrm{FNV})$ correction term. The $\mathrm{E}_{\mathrm{cor}}^{\mathrm{q}}$ accounts for the potential alignment between the charged defect and bulk at a point far from the defect. In addition, the $\mathrm{E}_{\mathrm{cor}}^{\mathrm{q}}$ accounts for the charge corrections in a supercell of finite size $[36,35]$. The FNV scheme explicitly uses the electrostatic potential obtained from DFT calculations to obtain an electrostatics model. The defect transition energy level $\epsilon\left(q / q^{\prime}\right)$ which is the Fermi energy at which two different charge states of the same defect have the same energy of formation, is given as [35]

$$
\epsilon\left(q / q^{\prime}\right)=\frac{E^{f}\left(\mathrm{RE}_{\mathrm{Ge}}, q ; \varepsilon_{F}=0\right)-E^{f}\left(\mathrm{RE}_{\mathrm{Ge}}, q^{\prime} ; \varepsilon_{F}=0\right)}{q^{\prime}-q}
$$

As reported in ref [2], we took the modelled band gap of the pristine Ge to be $0.78 \mathrm{eV}$.

\section{Results and Discussion}

\subsection{Structural properties}

Fig. 1 shows the relaxed geometric structures of RE substitutional impurities in Ge. Figs. 1a and 1b display relaxed geometric structures of the $\mathrm{Ce}_{\mathrm{Ge}}$ and $\operatorname{Pr}_{\mathrm{Ge}}$, respectively, and Figs. 1c and 1d display the relaxed geometric structures of the $\mathrm{Eu}_{\mathrm{Ge}}$ and $\mathrm{Er}_{\mathrm{Ge}}$, respectively. Table 1 lists the predicted shortest bond distance $\left(\beta_{\mathrm{d}}\right)$ in $(\AA)$ between a RE and its nearest neighbour Ge atom, and the difference $\Delta_{\mathrm{d}}$ between the $\beta_{\mathrm{d}}$ and the relaxed Ge-Ge bond length. After structural relaxation, we obtained the bond angle between three Ge atoms to be $109.4^{\circ}$ and Ge-Ge bond length to be $2.46 \AA$. 
This bond length is in close agreement with both experimental (2.45 $\AA$ ) [37] and GGA theoretical (2.48 $\AA$ ) [38] results. The shortest bond length between $\mathrm{Ce}, \mathrm{Pr}, \mathrm{Eu}, \mathrm{Er}$ and its nearest neighbour Ge atom is 2.80, 2.78, 2.74 and $3.18 \AA$, respectively. The difference between the pristine nearest neighbour Ge bond length and the RE-Ge bond lengths for the Ce, Pr, Eu and Er is 0.340 .320 .27 and $0.72 \AA$, respectively. The amount of strain in the bond length experienced by atoms plays a vital role in predicting the stability (using the formation energy) of any doped system. We observed that the order of increase of difference in the bond length of RE-Ge and that of Ge-Ge after structural relaxation is $\mathrm{Eu}<\mathrm{Ce}<\operatorname{Pr}<\operatorname{Er}$ (see Table 1). Based on this ordering, we expect the bond length of the Er-Ge to experience more strain than that of other dopants. This suggests that The energy required for Er-Ge to form due to the high strain experienced, will be higher compare to that of other RE-Ge. The bond angles formed by a RE and its nearest neighbour two Ge atoms for all the rare earth dopants in Ge being investigated are all approximately $109.5^{\circ}$. This suggests that there is no much difference between the bond angle of pristine Ge and that of the RE substitution in Ge.

\subsection{Total and projected density of states}

Fig. 2 displays the plot of both the total density of states (DOS) and projected density of states (PDOS) of Ge and the $\mathrm{RE}_{\mathrm{Ge}}$. For the pristine Ge, as shown in Figs. 2a and 2b, the majority (spin up) and minority (spin down) density of states are symmetrical, suggesting non-spin polarisation of the system. For the $\mathrm{Ce}_{\mathrm{Ge}}$ (Figs. 2c and 2d), $\operatorname{Pr}_{\mathrm{Ge}}$ (Figs. 2e and 2f) and $\mathrm{Eu}_{\mathrm{Ge}}$ (Figs. $2 \mathrm{~g}$ and $2 \mathrm{~h}$ ), the majority and minority spins are not symmetri- 
cal, which suggests that the ground state of these systems are spin polarised. But on the other hand, the ground state of the $\operatorname{Er}_{\mathrm{Ge}}$ (Figs. 2i and 2j) is not spin polarised since the spin up and spin down are symmetrically the same. For all $\mathrm{RE}_{\mathrm{Ge}}$, significant orbital states are located above the Fermi level at the VBM. For the $\mathrm{Ce}_{\mathrm{Ge}}$, the orbital states mainly contributed by the strong $p$-orbitals of both Ce and Ge atoms and $d$-orbital of Ce atoms, are 0.35 and $0.04 \mathrm{eV}$ above the Fermi level for the minority and majority spins, respectively. This suggests that the $\mathrm{Ce}_{\mathrm{Ge}}$ has a $p$-type semiconductor behaviour and can be considered for application in spintronics. The $\operatorname{Pr}_{\mathrm{Ge}}$ the orbital states contributed by $p$ and $d$-orbitals ground state of Pr atom are 0.34 and $0.02 \mathrm{eV}$ above the Fermi level for the spin down and up, respectively. For the $\mathrm{Eu}_{\mathrm{Ge}}$, the contribution of the $p$ and $d$-orbital states are 0.28 and $0.01 \mathrm{eV}$ above the Fermi level for the minority and majority spins, respectively. We also observed that for the $\operatorname{Er}_{\mathrm{Ge}}$, the contribution of both the $p$ and $d$-orbital states is located above the Fermi level at the valence band maximum with energy of $0.36 \mathrm{eV}$, suggesting a $p$-type semiconductor material. Apart from the contributions by the orbital states above the Fermi level at the VBM, we observed a strong orbital hybridization between the $p$ and $s$-orbitals of Ge. For the $\mathrm{Re}_{\mathrm{Ge}}$, (depending of the participating RE atom) we noticed orbital hybridisation between $p$-orbital and s-orbital of Ge and RE atoms, respectively. In addition, a noticeable significant hybridization occurs between $p$-orbitals of both participating RE and Ge atoms. Generally speaking, for all the $\operatorname{Re}_{\mathrm{Ge}}$ there is strong presence of each $\mathrm{RE} d$-orbital in the band gap of Ge. 


\subsection{Formation energies and charge state transition levels}

Table 2 lists the results of formation energies at $\epsilon_{f}=0$ for the neutral charge state of the $\mathrm{Ce}_{\mathrm{Ge}}, \operatorname{Pr}_{\mathrm{Ge}}, \mathrm{Eu}_{\mathrm{Ge}}$ and $\mathrm{Er}_{\mathrm{Ge}}$. As listed in Table 2, the formation energies of the $\mathrm{RE}_{\mathrm{Ge}}$ varied from -0.14 to $3.13 \mathrm{eV}$. For all $\mathrm{RE}_{\mathrm{Ge}}$, the energy of formation $(-0.14 \mathrm{eV})$ of the $\operatorname{Pr}_{\mathrm{Ge}}$ is the lowest and the $\operatorname{Er}_{\mathrm{Ge}}$ has the highest formation energy of $3.13 \mathrm{eV}$. For the neutral charge state, the energy of formation of the $\operatorname{Er}_{\mathrm{Ge}}$ is at least $3.00 \mathrm{eV}$ higher than the other dopants. This suggests that the Er as a dopant in Ge, under equilibrium conditions requires a higher formation energy to form compare to other RE (Ce, Eu and Pr) dopants in Ge. In addition, as already mentioned in the structural part, the high strain experienced by the atoms of $\operatorname{Er}_{\mathrm{Ge}}$ could also possibly be the reason while it has the highest formation energy. Considering that the strain experienced by the atoms of $\operatorname{Pr}_{\mathrm{Ge}}$ are less than that of $\operatorname{Er}_{\mathrm{Ge}}$ and $\mathrm{Ce}_{\mathrm{Ge}}$, and that the formation energy of the $\operatorname{Pr}_{\mathrm{Ge}}$ is lower than $\mathrm{Ce}_{\mathrm{Ge}}$ with $0.21 \mathrm{eV}$, hence: we expect the $\operatorname{Pr}_{\mathrm{Ge}}$ under equilibrium condition to be energetically the most favourable.

Plots of the formation energy as a function of the Fermi energy for the $\mathrm{RE}_{\mathrm{Ge}}$ are shown in Fig. 3. Table 3 lists the charge state transition levels. The induced charge state transition levels for the $\mathrm{Ce}_{\mathrm{Ge}}$ are deep within the band gap of Ge (see Fig. 3a). The first noticeable charge state transition level of the $\mathrm{Ce}_{\mathrm{Ge}}$ is $(+2 /+1)$, a deep donor lying at $0.21 \mathrm{eV}$ above the VBM. The $\mathrm{Ce}_{\mathrm{Ge}}$ also induced a $(+1 /-1)$ charge state thermodynamic transition level at $\mathrm{E}_{\mathrm{C}}-0.38 \mathrm{eV}$. The $(+1 /-1)$ charge state transition level induced by the $\mathrm{Ce}_{\mathrm{Ge}}$ exhibits properties of negative- $U$ ordering. By using the method of Refs $[18,19]$, we calculated the effective- $U$ value of the $(+1 /-1)$ negative- 
$U$ ordering to be $-0.82 \mathrm{eV}$, this suggests that the $\mathrm{Ce}_{\mathrm{Ge}}$ experiences large lattice distortion when charges are introduced. Fig. 3b shows that the $\operatorname{Pr}_{\mathrm{Ge}}$ induced a shallow $(-1 /-2)$ acceptor level with an energy of $0.10 \mathrm{eV}$ below the conduction band minimum. For the $\operatorname{Pr}_{\mathrm{Ge}}$, we also observed additional two charge state transition levels the $(+1 / 0)$ and $(+1 /-2)$ which are not thermodynamically stable. The $\operatorname{Pr}_{\mathrm{Ge}}$ did not exhibit any negative- $U$ ordering and donor level for all Fermi energies in the band gap of Ge as observed for the case of the $\mathrm{Ce}_{\mathrm{Ge}}$. The same charge state transition level observed for the $\operatorname{Pr}_{\mathrm{Ge}}$ is also observed for the $\mathrm{Eu}_{\mathrm{Ge}}$. The only difference is that for the $\mathrm{Eu}_{\mathrm{Ge}}$, the $(-1 /-2)$ acceptor level is $0.11 \mathrm{eV}$ below the conduction band as shown in Fig. 3c. For the $\operatorname{Er}_{\mathrm{Ge}}$, we found a shallow level at $(+1 /-1)$ in the band gap of Ge as displayed by Fig. 3d. The $\operatorname{Er}_{\mathrm{Ge}}$ exhibits a negative- $U$ ordering with effective- $U$ value of $-0.89 \mathrm{eV}$.

\section{Summary}

Results of the formation energies and charge state transition levels of the $\mathrm{Ce}, \mathrm{Pr}, \mathrm{Eu}$ and Er substitutions in Ge were described in detail. The energy of formation of the $\mathrm{Ce}_{\mathrm{Ge}}, \operatorname{Pr}_{\mathrm{Ge}} \mathrm{Er}_{\mathrm{Ge}}$ and $\mathrm{Eu}_{\mathrm{Ge}}$ for the neutral charge state lies between -0.14 and $3.13 \mathrm{eV}$. Amongst the RE, the formation energy of the $\operatorname{Pr}_{\mathrm{Ge}}$ for the neutral charge state is the lowest and energetically the most favourable. We have shown that the RE substitutional impurities induced charged state transition levels in the band gap of Ge. The $\mathrm{Ce}_{\mathrm{Ge}}$ induced a deep donor level at $\mathrm{E}_{\mathrm{V}}+0.38 \mathrm{eV}$ for the $(+2 /+1)$ charge states transition level. The $\mathrm{Pr}_{\mathrm{Ge}}$ and $\mathrm{Eu}_{\mathrm{Ge}}$ induced only acceptor levels within the band gap of Ge. For the $\mathrm{Eu}_{\mathrm{Ge}}$ and $\operatorname{Pr}_{\mathrm{Ge}}$, the induced levels are at $\mathrm{E}_{\mathrm{C}}-0.11$ and 
$\mathrm{E}_{\mathrm{C}}-0.10 \mathrm{eV}$, respectively. The $\mathrm{Ce}_{\mathrm{Ge}}$ and $\mathrm{Er}_{\mathrm{Ge}}$ exhibit properties of negative$U$ ordering with effective- $U$ values of -0.82 and $-0.89 \mathrm{eV}$, respectively.

\section{Acknowledgement}

This work is based on the research supported partly by National Research foundation (NRF) of South Africa (Grant specific unique reference number (UID) 98961). The opinions, findings and conclusion expressed are those of the authors and the NRF accepts no liability whatsoever in this regard.

[1] F.J. Morin, J.P. Maita, Phys. Rev. 94, 1525 (1954)

[2] E. Igumbor, C. Ouma, G. Webb, W. Meyer, Physica B: Condensed Matter 480, 191 (2016)

[3] E. Igumbor, R.E. Mapasha, W.E. Meyer, Journal of Electronic Materials pp. 1-8 (2016). URL http://dx.doi.org/10.1007/s11664-016-5026-z

[4] C.O. Chui, K. Gopalakrishnan, P.B. Griffin, J.D. Plummer, K.C. Saraswat, Applied physics letters 83(16), 3275 (2003)

[5] C.E. Lindberg, J.L. Hansen, P. Bomholt, A. Mesli, K.B. Nielsen, A.N. Larsen, L. Dobaczewski, Applied Physics Letters 87(17), 172103 (2005)

[6] M.L.W. Thewalt, D. Labrie, I.J. Booth, B.P. Clayman, E.C. Lightowlers, E.E. Haller, Physica B+ C 146(1), 47 (1987)

[7] H. Bracht, physica status solidi (a) 211(1), 109 (2014)

[8] E. Igumbor, R. Mapasha, R. Andrew, W. Meyer, Computational Condensed Matter 8, 31 (2016) 
[9] A. Chroneos, Journal of Applied Physics 107(7) (2010)

[10] E. Igumbor, W. Meyer, Materials Science in Semiconductor Processing 43, 129 (2016)

[11] P. Śpiewak, J. Vanhellemont, K.J. Kurzydłowski, Journal of Applied Physics 110(6), (2011)

[12] H. Tahini, A. Chroneos, R.W. Grimes, U. Schwingenschlgl, H. Bracht, Applied Physics Letters 99(7), 072112 (2011)

[13] A. Colder, P. Marie, T. Wojtowicz, P. Ruterana, S. Eimer, L. Méchin, K. Lorenz, U. Wahl, E. Alves, V. Matias, Superlattices and Microstructures 36(4), 713 (2004)

[14] B. Garrido, C. Garcia, S.Y. Seo, P. Pellegrino, D. Navarro-Urrios, N. Daldosso, L. Pavesi, F. Gourbilleau, R. Rizk, Physical Review B 76(24), 245308 (2007)

[15] A. Kanjilal, L. Rebohle, M. Voelskow, M. Helm, W. Skorupa, Journal of Applied Physics 107(2) (2010)

[16] R. Jones, Optical Materials 28(6), 718 (2006)

[17] S. Manna, N. Prtljaga, S. Das, N. Daldosso, S. Ray, L. Pavesi, Nanotechnology 23(6), 065702 (2012)

[18] E. Igumbor, K. Obodo, W.E. Meyer, Solid State Phenomena 242, 440 (2015) 
[19] Freysoldt, Christoph, Grabowski, Blazej, Hickel, Tilmann, J. Neugebauer, Kresse, Georg, Janotti, Anderson, V. de Walle, C. G., Rev. Mod. Phys. 86, 253 (2014)

[20] E. Igumbor, R.C. Andrew, W.E. Meyer, Journal of Electronic Materials (2016)

[21] V. Markevich, Materials Science in Semiconductor Processing 9(45), 589 (2006). Proceedings of Symposium T E-MRS 2006 Spring Meeting on Germanium based semiconductors from materials to devices

[22] G. Impellizzeri, S. Mirabella, M. Grimaldi, Applied Physics A 103(2), $323(2011)$

[23] G.S. Pomrenke, E. Silkowski, J.E. Colon, D.J. Topp, Y.K. Yeo, R.L. Hengehold, Journal of Applied Physics 71(4) (1992)

[24] Y. Miyata, Y. Nose, T. Yoshimura, A. Ashida, N. Fujimura, Journal of Crystal Growth 425, 158 (2015)

[25] H. Przybylinska, W. Jantsch, Y. Suprun-Belevitch, M. Stepikhova, L. Palmetshofer, G. Hendorfer, A. Kozanecki, R.J. Wilson, B.J. Sealy, Phys. Rev. B 54, 2532 (1996)

[26] J. Heyd, G.E. Scuseria, M. Ernzerhof, The Journal of Chemical Physics 118(18), 8207 (2003)

[27] G. Kresse, J. Furthmüller, Phys. Rev. B 54, 11169 (1996)

[28] P.E. Blochl, Phys. Rev. B 50, 17953 (1994) 
[29] J.P. Perdew, K. Burke, M. Ernzerhof, Phys. Rev. Lett. 77, 3865 (1996)

[30] P. Deák, B. Aradi, T. Frauenheim, E. Janzén, A. Gali, Phys. Rev. B 81, 153203 (2010)

[31] B.G. Janesko, T.M. Henderson, G.E. Scuseria, Physical Chemistry Chemical Physics 11(3), 443 (2009)

[32] J.L. Da Silva, M.V. Ganduglia-Pirovano, J. Sauer, V. Bayer, G. Kresse, Physical Review B 75(4), 045121 (2007)

[33] H.J. Monkhorst, J.D. Pack, Phys. Rev. B 13, 5188 (1976)

[34] S.B. Zhang, J.E. Northrup, Phys. Rev. Lett. 67, 2339 (1991)

[35] C. Freysoldt, J. Neugebauer, C.G. Van de Walle, physica status solidi (b) 248(5), 1067 (2011)

[36] C. Freysoldt, J. Neugebauer, C.G. Van de Walle, Phys. Rev. Lett. 102, $016402(2009)$

[37] D. Batchelder, R. Simmons, The Journal of Chemical Physics 41(8), $2324(1964)$

[38] A. Chroneos, B. Uberuaga, R. Grimes, Journal of Applied Physics 102(8), 083707 (2007) 


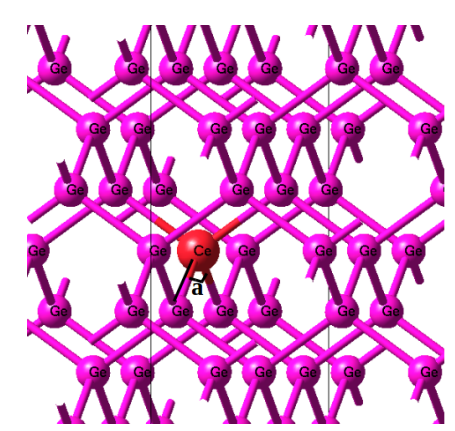

(a)

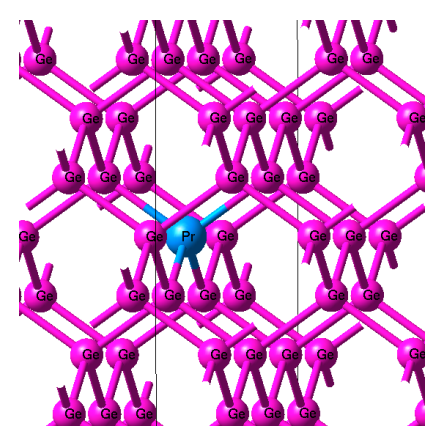

(b)

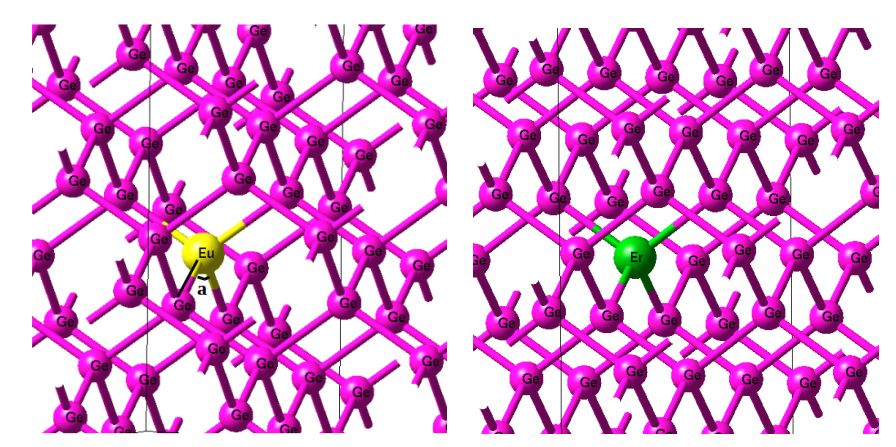

(c)

(d)

Figure 1: Relaxed geometric structures of RE substitutions in Ge. (a) $\mathrm{Ce}_{\mathrm{Ge}}$, (b) $\mathrm{Pr}_{\mathrm{Ge}}$, (c) $\mathrm{Eu}_{\mathrm{Ge}}$ and (d) $\mathrm{Er}_{\mathrm{Ge}}$. According to Figs. 1a and 1c, the black line and the text "a" represent the shortest bond length and the angle formed between a RE and two nearest neighbour Ge atoms. This same pattern applies to all other $\mathrm{RE}_{\mathrm{Ge}}$ systems. 


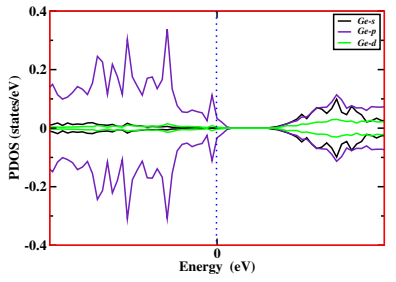

(a)

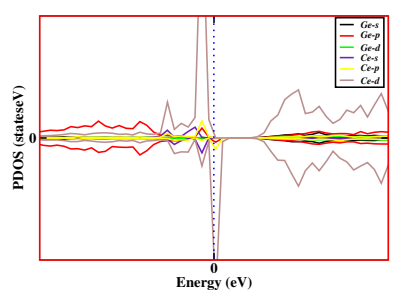

(c)

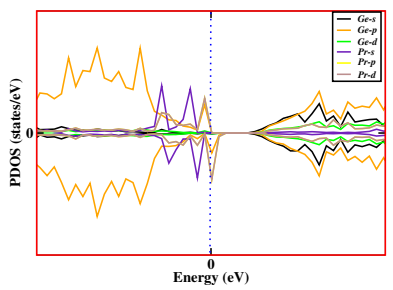

(e)

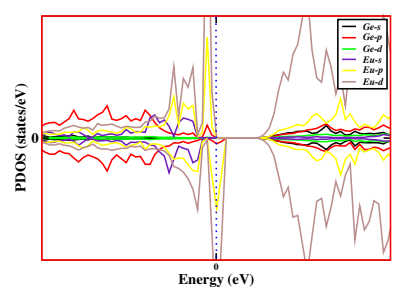

(g)

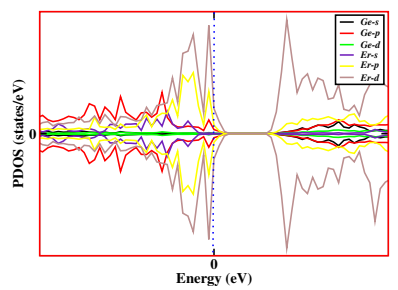

(i)

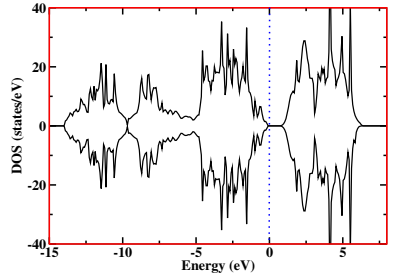

(b)

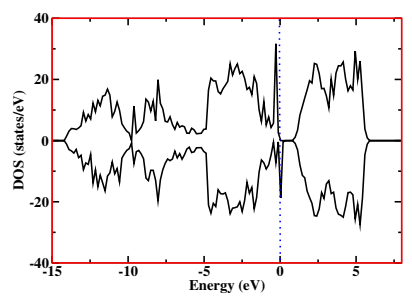

(d)

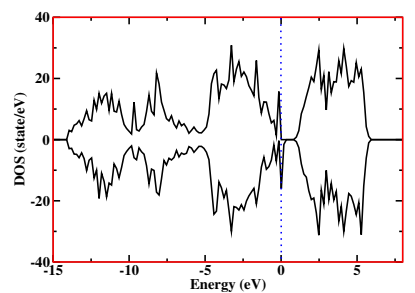

(f)

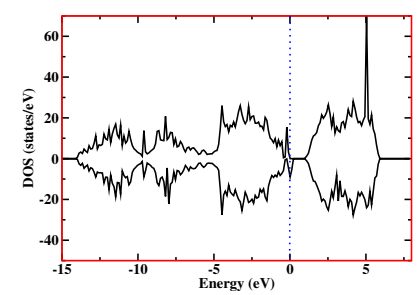

(h)

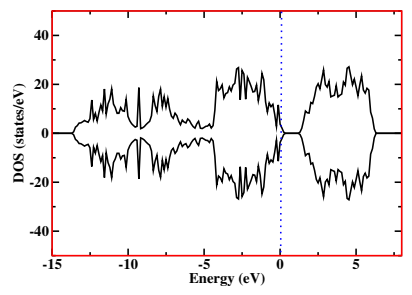

(j)

Figure 2: Plots showing the spin polarised partial density of states (PDOS) left and total density of states (DOS) right of the $\mathrm{RE}_{\mathrm{Ge}}$. The Fermi level (at $\epsilon_{f}=0 \mathrm{eV}$ ) is shown by the dashed vertical blue line. (a) PDOS of pristine Ge, (b) Total DOS of pristine Ge, (c) PDOS of $\mathrm{Ce}_{\mathrm{Ge}}$, (d) Total DOS of $\mathrm{Ce}_{\mathrm{Ge}}$, (e) PDOS of $\mathrm{Pr}_{\mathrm{Ge}}$, (f) Total DOS of $\mathrm{Pr}_{\mathrm{Ge}}$, (g) PDOS of $\mathrm{Eu}_{\mathrm{Ge}}$, (h) Total DOS of $\mathrm{Eu}_{\mathrm{Ge}}$, (i) PDOS of $\mathrm{Er}_{\mathrm{Ge}}$ and (j) Total DOS of $\mathrm{Er}_{\mathrm{Ge}}$. 


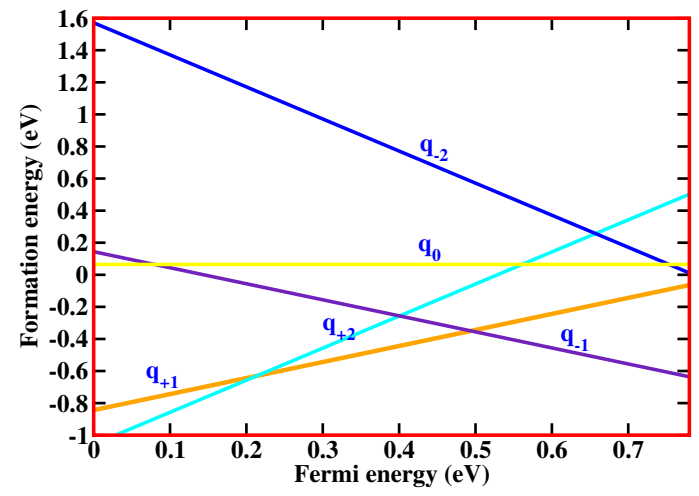

(a)

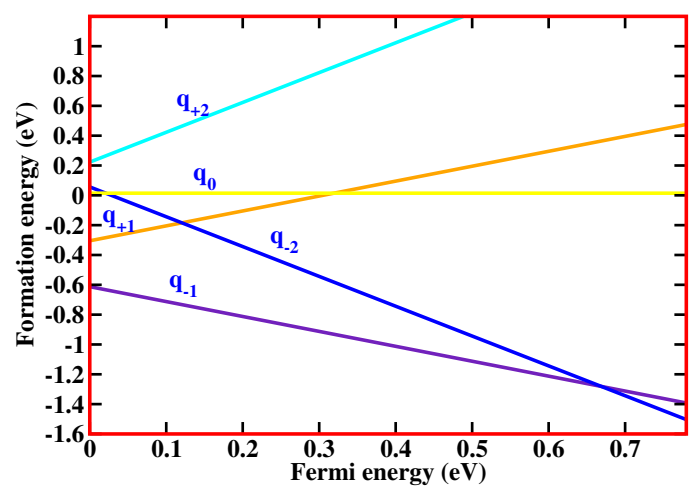

(c)

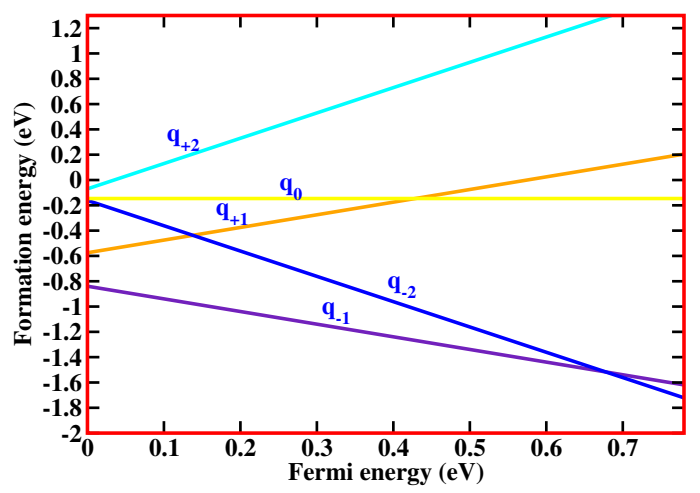

(b)

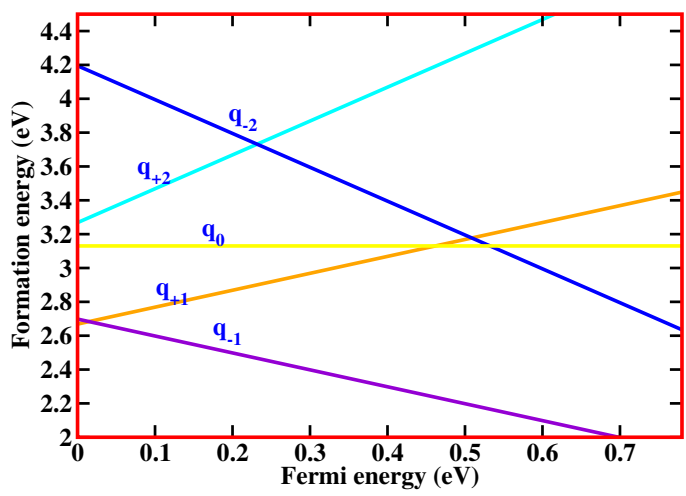

(d)

Figure 3: Plot of formation energy as a function of the Fermi energy for RE substitutional impurities in Ge; (a) The $\mathrm{Ce}_{\mathrm{Ge}}$ showing evidence of a donor level and negative-U properties in the band gap of Ge; (b) The $\operatorname{Pr}_{\mathrm{Ge}}$ showing evidence of an acceptor level in the band gap of Ge; (c) The Eu $\mathrm{u}_{\mathrm{Ge}}$ showing an acceptor level close to the conduction band. (d) The $\mathrm{Er}_{\mathrm{Ge}}$ showing a negative- $U$ ordering. 
Table 1: Predicted bond length $\left(\beta_{\mathrm{d}}\right)$ between a RE and Ge atoms after geometric relaxation and the difference $\left(\Delta_{\mathrm{d}}\right)$ between $\beta_{\mathrm{d}}$ and $\mathrm{Ge}-\mathrm{Ge}$ relaxed bond length $(2.46 \AA)$ for $\mathrm{RE}$ substitutional impurities in Ge. The RE-Ge bond length is calculated with respect to the nearest neighbour Ge atoms around the RE.

\begin{tabular}{ccc} 
& $\beta_{\mathrm{d}}(\AA)$ & $\Delta_{\mathrm{d}}(\AA)$ \\
\hline $\mathrm{Ce}-\mathrm{Ge}$ & 2.80 & 0.34 \\
$\mathrm{Pr}-\mathrm{Ge}$ & 2.78 & 0.32 \\
$\mathrm{Eu}-\mathrm{Ge}$ & 2.73 & 0.27 \\
$\mathrm{Er}-\mathrm{Ge}$ & 3.18 & 0.72 \\
\hline
\end{tabular}

Table 2: Calculated formation energies $\left(E^{f}\right)$ in $\mathrm{eV}$ at $\epsilon_{f}=0$ for the neutral charge state of the $\mathrm{Ce}_{\mathrm{Ge}}, \mathrm{Eu}_{\mathrm{Ge}} \mathrm{Er}_{\mathrm{Ge}}$ and $\operatorname{Pr}_{\mathrm{Ge}}$. The difference in formation energy $\left(d E^{f}\right)$ was calculated with respect to the lowest formation energy.

\begin{tabular}{ccc} 
Defect & $E^{f}$ & $d E^{f}$ \\
\hline $\mathrm{Ce}$ & 0.07 & 0.21 \\
$\mathrm{Er}$ & 3.13 & 3.27 \\
$\mathrm{Eu}$ & 0.01 & 0.15 \\
$\mathrm{Pr}$ & -0.14 & 0.00 \\
\hline
\end{tabular}

Table 3: The energy of the charge state transition levels $\epsilon\left(q / q^{\prime}\right)$ in $\mathrm{eV}$ within the band gap of Ge for the $\mathrm{Ce}_{\mathrm{Ge}}, \mathrm{Eu}_{\mathrm{Ge}} \mathrm{Er}_{\mathrm{Ge}}$ and $\operatorname{Pr}_{\mathrm{Ge}}$.

\begin{tabular}{lllll}
\hline Transition level & $\mathrm{Ce}_{\mathrm{Ge}}$ & $\mathrm{Eu}_{\mathrm{Ge}}$ & $\mathrm{Er}_{\mathrm{Ge}}$ & $\operatorname{Pr}_{\mathrm{Ge}}$ \\
\hline$(+2 /+1)$ & $\mathrm{E}_{\mathrm{V}}+0.21$ & - & - & - \\
$(-1 /-2)$ & - & $\mathrm{E}_{\mathrm{C}}-0.11$ & - & $\mathrm{E}_{\mathrm{C}}-0.10$ \\
$(+2 /-1)$ & $\mathrm{E}_{\mathrm{C}}-0.38$ & - & & - \\
$(+1 /-1)$ & - & - & $\mathrm{E}_{\mathrm{V}}+0.02$ & - \\
\hline
\end{tabular}

